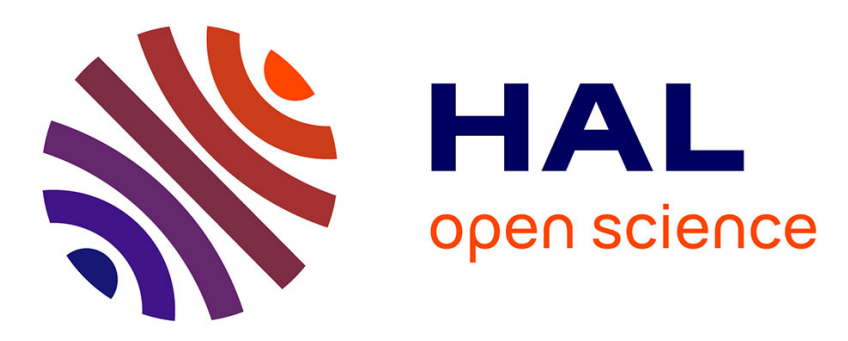

\title{
Ghost solutions with centered schemes for one-dimensional transport equations with Neumann boundary conditions
}

\author{
Mélanie Inglard, Frédéric Lagoutière, Hans Henrik Rugh
}

\section{To cite this version:}

Mélanie Inglard, Frédéric Lagoutière, Hans Henrik Rugh. Ghost solutions with centered schemes for one-dimensional transport equations with Neumann boundary conditions. Annales de la Faculté des Sciences de Toulouse. Mathématiques., In press, 10.5802/afst.1650 . hal-01899624

\section{HAL Id: hal-01899624 \\ https://hal.science/hal-01899624}

Submitted on 19 Oct 2018

HAL is a multi-disciplinary open access archive for the deposit and dissemination of scientific research documents, whether they are published or not. The documents may come from teaching and research institutions in France or abroad, or from public or private research centers.
L'archive ouverte pluridisciplinaire HAL, est destinée au dépôt et à la diffusion de documents scientifiques de niveau recherche, publiés ou non, émanant des établissements d'enseignement et de recherche français ou étrangers, des laboratoires publics ou privés. 


\title{
Ghost solutions with centered schemes for one-dimensional transport equations with Neumann boundary conditions
}

\author{
Mélanie Inglard; Frédéric Lagoutière†and Hans Henrik Rugh ${ }^{\ddagger}$
}

October 19, 2018

\section{Introduction}

The transport equation with constant velocity in an interval is essentially trivial from a mathematical point of view and does not a priori merit much academic consideration. Through numerical simulations, however, we came across a rather astonishing and apparently unknown phenomenon involving "ghost" solutions which we are going to describe in the following. To be more specific, consider the transport equation with constant velocity $a$ on the interval $(0,1)$. As numerical approximation we use a space-centered $\theta$-scheme. It is known (see [GKO95]) that on the whole real line, the $\theta$-scheme is stable in a strong sense in $l^{2}$ for $\theta \geq 1 / 2$ (it makes the $l^{2}$ norm of the solution non-increase at every time step). For $\theta \in[0,1 / 2)$, it can lead to a stable (in the sense that the $l^{2}$ norm can increase in a bounded way) and convergent scheme, but under a stability condition asking $\Delta t$ to be of the order of $\Delta x^{2}$, which makes the scheme less interesting. In the following, we consider $\theta \geq 1 / 2$.

A first difficulty comes from the fact that the centered scheme requires two boundary conditions (one for each boundary). The study of finite difference schemes for initial boundary value problems has a long story. The transport equation on the half line with Dirichlet boundary conditions has been studied, for dissipative schemes, by Kreiss and Lundqvist in [KL68], but the present scheme is not dissipative in their sense, and we here consider the equation on an intervall. Dirichlet boundary conditions at an outflow boundary often create boundary layers (see [KL68], and also [BC17] for their precise study), and a lot of papers have been devoted to avoid these boundary layers, to "let the solution go outside of the domain". Among these methods, let us cite the transparent boundary conditions (see the review paper $\left[\mathrm{AAB}^{+} 08\right]$ ) and the absorbing boundary conditions (see [Ehr10]). A common way to "let the solution go out" is to provide the scheme with artificial (numerical) homogeneous Neumann boundary conditions (which is especially useful when dealing with non-linear systems), and this is the center of the present paper.

We consider here two ways to provide the scheme with boundary conditions: First, we propose homogeneous Neumann conditions on both sides and observe the appearance of "ghost" solutions with a periodic behavior (see section 2). On the continuous level the Neumann conditions correspond to the problem

$$
\left\{\begin{array}{l}
\partial_{t} u+a \partial_{x} u=0, \quad t \in \mathbb{R}_{+}, \quad x \in(0,1), \\
\partial_{x} u(t, 0)=0, \quad t \in \mathbb{R}_{+}, \\
\partial_{x} u(t, 1)=0, \quad t \in \mathbb{R}_{+}, \\
u(0, x)=u^{0}(x), \quad x \in(0,1) .
\end{array}\right.
$$

This is known to be ill-posed in $L^{2}$, what is easy to understand: with initial condition $u^{0}=0$, the function $u(t, x)=0$ is a solution to the problem. But taking a perturbation of the initial condition of the form $u_{\epsilon}^{0}(x)=\chi_{(0, \epsilon)}(x)$, for $\epsilon \in(0,1)$ one observes that $u_{\epsilon}(t, x)=\chi_{(0, \epsilon+a t)}(x)$ is a solution for $t<(1-\epsilon) / a$. Thus $\left\|u^{0}-u_{\epsilon}^{0}\right\|_{2}=\epsilon^{1 / 2}$, but $\left\|u(t, \cdot)-u_{\epsilon}(t, \cdot)\right\|_{2}=(\epsilon+a t)^{1 / 2}$, which implies the lack of continuous dependence in $L^{\infty}\left(0,(1-\epsilon) / a, L^{2}(0,1)\right)$ (take $\epsilon$ small and $\left.a>0\right)$.

\footnotetext{
${ }^{*}$ Lycée Saint-Louis, 44 boulevard Saint-Michel, 75006 Paris, France. Email: melinglard@gmail.com.

†Université de Lyon, Université Claude Bernard Lyon 1, Institut Camille Jordan (CNRS UMR5208), 43 boulevard du 11 novembre 1918, F-69622 Villeurbanne Cedex, France. Email: lagoutiere@math.univ-lyon1.fr. Research of the author was supported by ANR project BoND, ANR-13-BS01-0009, and by ANR project NABUCO, ANR-17-CE40-0025.

${ }^{\ddagger}$ Laboratoire de Mathématiques d’Orsay, Univ. Paris-Sud, CNRS, Université Paris-Saclay, 91405 Orsay, France. Email: Hans-Henrik.Rugh@math.u-psud.fr.
} 
Second, we analyze the case of homogeneous Dirichlet conditions on both sides and show another strange periodical behavior, also involving "ghost" solutions.

Our purpose here is not to discuss whether or not Neumann (or Dirichlet) boundary conditions are legitimate: they are not, in the strong sense. Nevertheless, they are natural conditions when performing numerical simulations and the "ghost" phenomenon that we describe seems robust and could turn out to be a general phenomenon in similar numerical situations even for more complex nonlinear hyperbolic systems (for which it is a rather usual cheap way to impose numerical homogeneous Neumann conditions, as the correct ones are quite hard to know). Indeed, similar experiments to those in the present paper but performed for the non-linear scalar Burgers' equation, as well as for the Euler system of compressible gas dynamics in dimension 1 exhibit the same strange characteristics.

The paper is organized as follows. In section 2, we present the $\theta$-scheme with Neumann boundary conditions, and the peculiar numerical results that motivated the present study, and we formulate a convergence theorem. In section 3, we prove the theorem. Then, in section 4 , we state a similar result in the case where (homogeneous) Dirichlet boundary conditions are put on both sides. Finally, some numerical results for various situations are reported in section 5 .

\section{The $\theta$-scheme for the one-dimensional transport equation with Neumann boundary conditions}

For a given (smooth) initial condition $u_{0}$, we consider Problem (1) where $a$ is a given real parameter. Let $J \in \mathbb{N} \backslash\{0\}$ be the number of cells in $[0,1], \Delta x=1 / J$ their length, and $\Delta t>0$. We define as $x_{j}=(j-1 / 2) \Delta x$ the center of the cell number $j, j \in\{1, \ldots, J\}$. Let $\theta \geq 0$. The centered $\theta$-scheme for the above initial boundary value problem is

$$
\left\{\begin{array}{l}
\frac{U_{j}^{n+1}-U_{j}^{n}}{\Delta t}+a \theta \frac{U_{j+1}^{n+1}-U_{j-1}^{n+1}}{2 \Delta x}+a(1-\theta) \frac{U_{j+1}^{n}-U_{j-1}^{n}}{2 \Delta x}=0, \quad n \geq 0, \quad 2 \leq j \leq J-1, \\
\frac{U_{1}^{n+1}-U_{1}^{n}}{\Delta t}+a \theta \frac{U_{2}^{n+1}-U_{1}^{n+1}}{2 \Delta x}+a(1-\theta) \frac{U_{2}^{n}-U_{1}^{n}}{2 \Delta x}=0, \quad n \geq 0, \\
\frac{U_{J}^{n+1}-U_{J}^{n}}{\Delta t}+a \theta \frac{U_{J}^{n+1}-U_{J-1}^{n+1}}{2 \Delta x}+a(1-\theta) \frac{U_{J}^{n+1}-U_{J-1}^{n+1}}{2 \Delta x}=0, \quad n \geq 0, \\
U_{j}^{0}=u^{0}\left(x_{j}\right), \quad j \in\{1, \ldots, J\} .
\end{array}\right.
$$

The second and the third equation above can be obtained from the first by defining the fictive boundary values $U_{0}^{n}=U_{1}^{n}$ and $U_{J+1}^{n}=U_{J}^{n}$ for every $n$. Note that this is consistent with homogeneous Neumann conditions.

In the sequel we will often make use of two versions of $J$ by $J$ "difference matrices". The first corresponds to Neumann boundary conditions, the second to periodic boundary conditions:

$$
D_{J}=\left(\begin{array}{cccccc}
-1 & 1 & 0 & \cdots & \cdots & 0 \\
-1 & 0 & 1 & & & \vdots \\
\vdots & & \ddots & \ddots & & \vdots \\
\vdots & & & -1 & 0 & 1 \\
0 & \cdots & \cdots & 0 & -1 & 1
\end{array}\right) \text { and } T_{J}=\left(\begin{array}{cccccc}
0 & 1 & 0 & \cdots & \cdots & -1 \\
-1 & 0 & 1 & & & \vdots \\
\vdots & & \ddots & \ddots & & \vdots \\
\vdots & \cdots & & -1 & 0 & 1 \\
1 & \cdots & \cdots & 0 & -1 & 0
\end{array}\right)
$$

both matrices belonging to $\mathcal{M}_{J}(\mathbb{R})$. We shall often omit the subscript $J$ in order to ease notation. Set $U^{n}=\left(U_{j}^{n}\right)_{j=1}^{J} \in \mathbb{R}^{J}$ and denote by $c=a \Delta t / \Delta x$ the Courant number, The matrix form of the scheme (2) is then given by:

$$
A U^{n+1}+B U^{n}=0
$$

where $A=I+\frac{\theta c}{2} D, B=-I+\frac{(1-\theta) c}{2} D$ and $I$ is the $J \times J$ identity matrix.

To see that this scheme always has a solution it suffices to show that $d_{J}(z)=\operatorname{det}\left(1-s D_{J}\right)$ has no real roots. Developing e.g. from the top left one shows that $d_{J+2}(z)=d_{J+1}(z)+z^{2} d_{J}(z), J \geq 2$. As $d_{2}(z)=1$ and $d_{3}(z)=1+z^{2}$ it follows that every $d_{J}(z), J \geq 2$ is a polynomial in $z^{2}$ with only positive coefficients so indeed there can be no real roots. 
Figures 1 and 2 shows some results obtained with $a=1, \theta=1$ (the implicit scheme), and $c=1$, for the $C^{\infty}$ initial condition

$$
u^{0}(x)=\left\{\begin{array}{l}
\frac{1}{\exp (-9)} \exp \left(\frac{1}{(x-1 / 2)^{2}-\frac{1}{9}}\right) \text { if }\left|x-\frac{1}{2}\right|<\frac{1}{3}, \\
0 \text { if } x \in(0,1) \backslash(1 / 6,5 / 6)
\end{array}\right.
$$

with a number of cell that is even or odd.
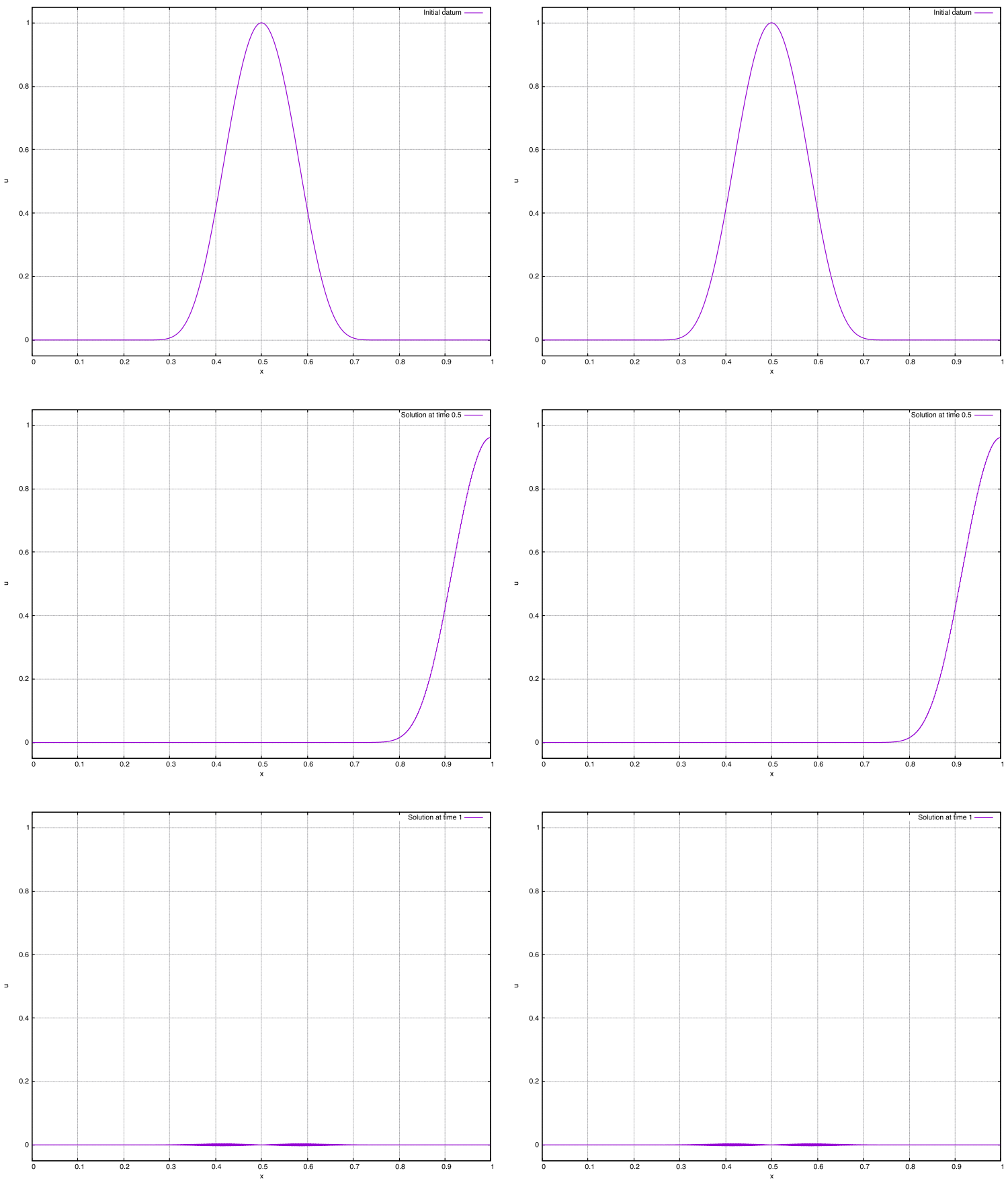

Figure 1: Numerical results: with 1000 cells on the left, 1001 cells on the right. From top to bottom: time $0,0.5,1$. 

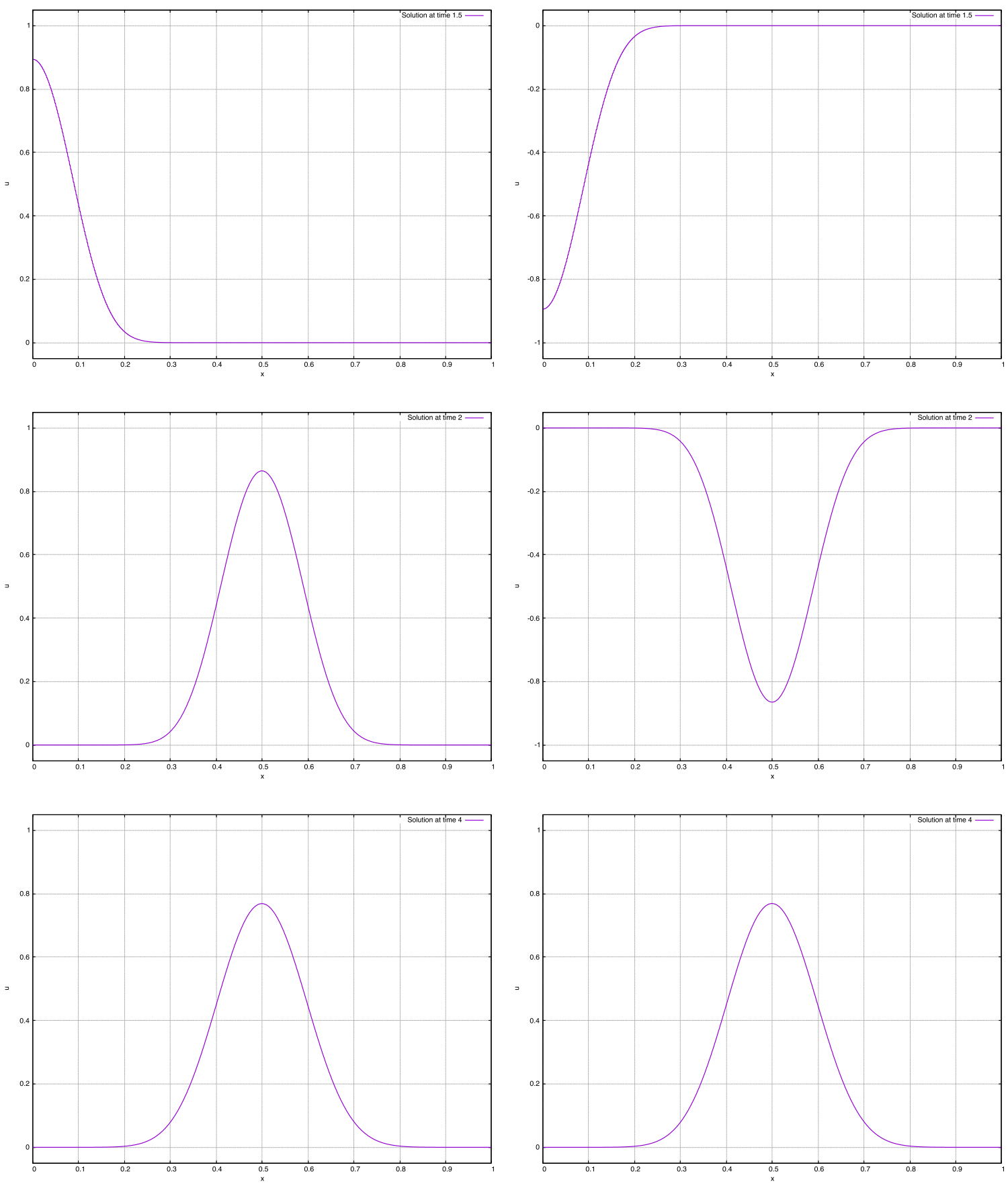

Figure 2: Numerical results: with 1000 cells on the left, 1001 cells on the right. From top to bottom: time $1.5,2,4$.

Let us comment upon the observed numerical solutions. At the beginning of the computation (until time 1), the results are as "expected": the profile translates to the right, and the numerical Neumann boundary conditions behave "normally" and resembles a transparency condition, i.e. the profile seems to go out of $(0,1)$. But then, with 1000 cells, the initial condition re-enters the domain from the left boundary, whereas, with 1001 cells, the opposite of the initial condition re-enters. At time 2, the solution with 1000 cells coincides (almost exactly) with the initial condition In fact, one observes numerically that the solution at time 2 converges toward the initial condition as the number of cells, $J$, tends to $\infty$ taking even values only. On the other hand, the solution with 1001 cells coincides (almost exactly) with the opposite of the initial condition. The ongoing process continues 
periodically, so that at time $T=4$ one recovers in both cases the (almost exact) initial conditon. The period is $T=4 / a$ for a general velocity $a$.

The above observations have led us to formulate a theorem as follows: We extend the initial condition to all of $\mathbb{R}$ depending on the parity of the number of cells, i.e. we define the functions $u^{0, e}$ (e for even) and $u^{0, o}$ (o for odd) on $\mathbb{R}$ by :

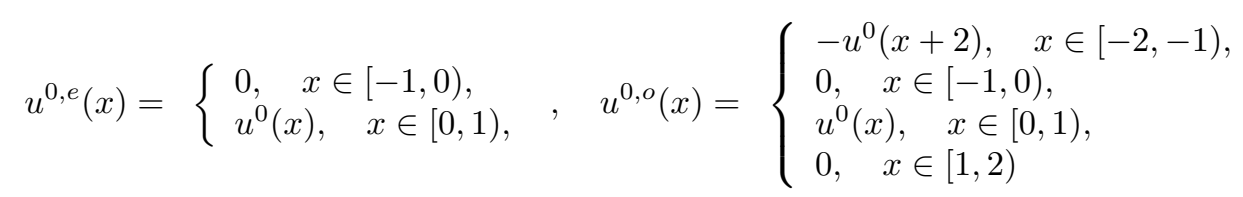

and we require $u^{0, e}$ to be 2-periodic and $u^{0, o}$ to be 4 -periodic. Let $u^{*}$ be the unique (a fortiori 2- or 4 -periodical) solution to the transport problem on the full real line:

$$
\left\{\begin{array}{l}
\partial_{t} u+a \partial_{x} u=0, \quad t \in \mathbb{R}_{+}, \quad x \in \mathbb{R} \\
u(0, x)=u^{0, *}(x)
\end{array}\right.
$$

with $u^{0, *}=u^{0, e}$ or $u^{0, o}$. Also define $e_{j}^{*, n}=u_{j}^{n}-u^{*}\left(t^{n}, x_{j}\right)$ for $j \in\{1, \ldots J\}, n \in \mathbb{N}\left(\operatorname{defining} t^{n}=n \Delta t\right)$. Finally, for $u=\left(u_{j}\right)_{j=1}^{J}$, write $|u|_{2}=\left(\sum_{j=1}^{J} \Delta x u_{j}^{2}\right)^{1 / 2}$ for the discrete $l^{2}(0,1)$ norm. Although it depends on $J$, we drop this dependence in the notation.

Theorem 2.1 Assume $u^{0} \in \mathcal{C}_{c}^{2}(0,1)$ and $u^{0}\left(x_{1}\right)=u^{0}\left(x_{J}\right)=0$. Then, there exists a constant $C$ such that, for any Courant number $c$ and any parameter $\theta \geq 1 / 2$, for all $N>0$, one has for $\Delta x$ small enough,

$$
\sup _{n \leq N}\left(\left|e^{n}\right|_{2}\right) \leq C(1+N \Delta t)\left(\Delta t+\Delta x^{1 / 2}\right) .
$$

Remark As $u^{0}$ is assumed to have compact support the condition $u^{0}\left(x_{1}\right)=u^{0}\left(x_{J}\right)$ is satisfied whenever $\Delta x$ is sufficiently small.

The idea of the proof is:

- First, we create a numerical (discrete) initial condition $\tilde{U}^{0}$ defined on $\{-J+1, \ldots J\}$ if $\mathrm{J}$ is even and on $\{-2 J+1, \ldots, 2 J\}$ if $\mathrm{J}$ is odd, in such a manner that the numerical solution $U^{n}$ is equal to the restriction on $\{1, \ldots, J\}$ of the numerical solution $\left(\tilde{U}^{n}\right)$ given by the scheme with periodical boundary condition,

- Second, we prove that the numerical solution with periodical boundary conditions converges (toward the restriction of $u^{*}$ on a period, $(-1,1)$ or $(-2,2)$ ). This part of the proof is classical and is a straight-forward application of the Lax-Richtmyer theorem: the scheme with these conditions will be shown to be stable and consistent.

\section{Proof of Theorem 2.1}

\subsection{Step 1: construction of a numerical (discrete) initial condition on a larger interval}

Ideally, we would like to analyse the scheme on the whole line $\mathbb{R}$, i.e. $\mathbb{Z}$ on the numerical level, by means of stability and consistency. We may indeed define an initial condition on $\mathbb{Z}$ such that the restriction to $\{1, \ldots, J\}$ of the numerical solution over $\mathbb{Z}$ equals the solution of the scheme on $\{1, \ldots, J\}$ with the homogeneous Neumann boundary conditions. When looking at the numerical results, however, such a reconstructed initial condition on $\mathbb{Z}$ must be 2- or 4 - periodic (or almost periodic). In particular, it will not belong to $l^{2}(\mathbb{Z})$, preventing us from doing a convergence analysis in $l^{2}(\mathbb{Z})$. Thus, we will slightly modify this program, and instead construct an initial condition on $\{-J+1, \ldots, J\}$ for $\mathrm{J}$ even and on $\{-2 J+1, \ldots, 2 J\}$ for $\mathrm{J}$ odd and consider the scheme on these sets with periodic boundary conditions. The restriction to $\{1, \ldots, J\}$ yields the wanted solution and the convergence analysis is easy in the periodic context.

We denote by $R$ the reconstruction operator. In the case where $J$ is even, it is an operator $R: \mathbb{R}^{J} \rightarrow \mathbb{R}^{2 J}$, and if $J$ is odd, it is of the form $R: \mathbb{R}^{J} \rightarrow \mathbb{R}^{4 J}$. 
Definition 3.1 The operator $R$ is defined as follows. Given $U=\left(U_{1}, \ldots, U_{J}\right) \in \mathbb{R}^{J}$ :

When $J$ is even, $u=\left(u_{-J+1}, \ldots, u_{J}\right)=R U \in \mathbb{R}^{2 J}$ is the unique vector such that

(a) $u_{j}=U_{j}$ for $j \in\{1, \ldots, J\}$,

(b) $u_{-j+1}-u_{-j}=(-1)^{j+1}\left(u_{j+1}-u_{j}\right)$ for $j \in\{0, \ldots, J-1\}$.

When $J$ is odd, $u=\left(u_{-3 J+1}, \ldots, u_{J}\right)=R U \in \mathbb{R}^{4 J}$ is the unique vector such that (a) and (b) hold, and also:

(c) $u_{-J-j+1}-u_{-J-j}=(-1)^{j+1}\left(u_{-J+j+1}-u_{-J+j}\right)$ for $j \in\{0, \ldots, J-1\}$,

(d) $u_{-2 J-j+1}-u_{-2 J-j}=(-1)^{j+1}\left(u_{-2 J+j+1}-u_{-2 J+j}\right)$ for $j \in\{0, \ldots, J-1\}$.

Remark 3.2 Note that the above defines $u$ by recursion, e.g. with $j=0$ in (b) one gets $u_{1}-u_{0}=$ $(-1)\left(u_{1}-u_{0}\right)$ or, equivalently, $u_{0}=u_{1}$ which precisely corresponds to the homogeneous Neumann condition. Then for $j=1$ we get $u_{0}-u_{-1}=(+1)\left(u_{2}-u_{1}\right)$ from which $u_{-1}=-u_{2}+u_{0}+u_{1}=-u_{2}+2 u_{1}$, etc. In the odd case, also note that (c) and (d) for $j=0$ imply: $u_{-J}=u_{-J+1}$ and $u_{-2 J}=u_{-2 J+1}$.

The utility of the reconstruction operator will become apparent from the proposition below. In order to simplify notation and unify our expressions for $J$ odd and even, let us introduce a vector $\hat{\alpha}(u)$ which for $J$ odd will just be the zero vector in $\mathbb{R}^{4 J}$ but for $J$ even and $u=R U \in \mathbb{R}^{2 J}$ is given by:

$$
\hat{\alpha}(u)=\left(\begin{array}{c}
\alpha(u) \\
0 \\
\vdots \\
0 \\
\alpha(u)
\end{array}\right) \in \mathbb{R}^{2 J}, \quad \text { with } \alpha(u)=\frac{c}{2}\left(u_{-J+1}-u_{J}\right) .
$$

Proposition 3.3 We have $\left\{\begin{array}{l}A V+B U=0, \\ v=R V,\end{array}\right.$ if and only if $\quad\left\{\begin{array}{l}u=R U, \\ P v+Q u=\hat{\alpha}(u) .\end{array}\right.$

Here,

$$
P=I+\frac{\theta c}{2} T \text { and } Q=-I+\frac{(1-\theta) c}{2} T,
$$

with $T$ being the (periodic) difference matrix from (3). For the sizes of vectors and matrices: If $J$ is even, $u, v \in \mathbb{R}^{2 J}$ and $P, Q \in \mathcal{M}_{2 J}(\mathbb{R})$, while if $J$ is odd, $u, v \in \mathbb{R}^{4 J}$ and $P, Q \in \mathcal{M}_{4 J}(\mathbb{R})$.

The above property expresses the fact that if we reconstruct the initial condition and work with the periodical scheme then $U^{n+1}$ solution of (2) will be the restriction to $\{1, \ldots, J\}$ of $u^{n+1}$, being a solution of

$$
P u^{n+1}+Q u^{n}=\hat{\alpha}\left(u^{0}\right) .
$$

The interest is that the convergence of this extended scheme is easy to obtain by the usual consistency and stability analysis. The second hand term above will be shown to be small if the initial data is twice continuously differentiable, and equal to 0 if e.g. it is symmetric with respect to $x=1 / 2$.

The proof of Proposition 3.3 will make use of the following lemma:

Lemma 3.4 For $J$ odd, and denoting $u=R U$, we have for $j \in\{0, \ldots, J-1\}$ :

(i) $u_{-2 J+j+1}-u_{-2 J+j}=-\left(u_{j+1}-u_{j}\right)$,

(ii) $u_{-3 J+j+1}-u_{-3 J+j}=-\left(u_{-J+j+1}-u_{-J+j}\right)$

(iii) $u_{-2 J+j}-u_{-J+1}=u_{J}-u_{j}$.

and also for $j \in\{1, \ldots, J-1\}$ :

(iv) $u_{-3 J+j+1}-u_{-3 J+j}=(-1)^{j+1}\left(u_{J-j+1}-u_{J-j}\right)$, 
Proof: The identities come from combining in different ways (a)-(d) from Definition 3.1. To see (i) we use (b) and substitute $J-j$ for $j$ in (c) to obtain: $u_{j+1}-u_{j}=$

$$
(-1)^{j+1}\left(u_{-j+1}-u_{-j}\right)=(-1)^{j+1}(-1)^{J-j+1}\left(u_{-2 J+j+1}-u_{-2 J+j}\right)=-\left(u_{-2 J+j+1}-u_{-2 J+j}\right) .
$$

Similarly, combining (c) and (d) we get (ii) and (iii) comes from (i) by summing from $j$ to $J-1$. Finally (iv) results from (b),(c) and (d) together.

Proof of Proposition 3.3: Consider first the case when $\mathrm{J}$ is even. Given $U \in \mathbb{R}^{J}, u=R U, V$ is obtained from $U$ by $A V+B U=0$, and $v$ is obtained from $u$ using $P v+Q u=(\alpha(u), 0, \ldots, 0, \alpha(u))^{t}$. We denote by $w=R V$ the reconstruction from $V$ and will show that $w=v$. We begin by proving that for any $j \in\{-J+1, \ldots, J\}$ we have

$$
w_{j}-u_{j}+\frac{\theta c}{2}\left(w_{j+1}-w_{j-1}\right)+\frac{(1-\theta) c}{2}\left(u_{j+1}-u_{j-1}\right)=0,
$$

provided we define $u_{-J}=u_{-J+1}, u_{J+1}=u_{J}$ and $w_{-J}=w_{-J+1}, w_{J+1}=w_{J}$. We already know the result for $j \in\{1, \ldots, J\}$ (since $R$ is the identity operator for that range of indices) and we proceed by induction.

- We have:

$$
\begin{aligned}
w_{0}=w_{1} & =u_{1}-\frac{\theta c}{2}\left(w_{2}-w_{1}\right)-\frac{(1-\theta) c}{2}\left(u_{2}-u_{1}\right) \\
& =u_{1}-\frac{\theta c}{2}\left(w_{0}-w_{-1}\right)-\frac{(1-\theta) c}{2}\left(u_{0}-u_{-1}\right) \\
& =u_{0}-\frac{\theta c}{2}\left(w_{1}-w_{-1}\right)-\frac{(1-\theta c)}{2}\left(u_{1}-u_{-1}\right),
\end{aligned}
$$

which proves (9) for $j=0$.

- We assume the result is proved for $j \in\{-k+1, \ldots, J\}$ (for $k \in 1, \ldots, J-1$ ). We have

$$
\begin{aligned}
w_{-k}= & w_{-k+1}+(-1)^{k+1}\left(w_{k}-w_{k+1}\right) \\
= & u_{-k+1}-\frac{\theta c}{2}\left(w_{-k+2}-w_{-k}\right)-\frac{(1-\theta) c}{2}\left(u_{-k+2}-u_{-k}\right)+(-1)^{k+1}\left(w_{k}-w_{k+1}\right) \\
= & u_{-k+1}-\frac{\theta c}{2}(-1)^{k}\left(w_{k}-w_{k-1}-\left(w_{k+1}-w_{k}\right)\right)-\frac{(1-\theta) c}{2}(-1)^{k}\left(u_{k}-u_{k-1}-\left(u_{k+1}-u_{k}\right)\right) \\
& +(-1)^{k+1}\left(w_{k}-w_{k+1}\right) \\
= & u_{-k+1}-\frac{\theta c}{2}(-1)^{k+1}\left(w_{k+1}-2 w_{k}+w_{k-1}\right)-\frac{(1-\theta) c}{2}(-1)^{k+1}\left(u_{k+1}-2 u_{k}+u_{k-1}\right)+(-1)^{k+1}\left(w_{k}-w_{k+1}\right)
\end{aligned}
$$

Therefore, we deduce

$$
\begin{aligned}
& w_{-k}+\frac{\theta c}{2}\left(w_{-k+1}-w_{-k-1}\right)+\frac{(1-\theta) c}{2}\left(u_{-k+1}-u_{-k-1}\right) \\
& =u_{-k+1}-\frac{\theta c}{2}(-1)^{k+1}\left(\left(w_{k-1}-w_{k}\right)-\left(w_{k+1}-w_{k+2}\right)\right) \\
& -\frac{(1-\theta c)}{2}(-1)^{k+1}\left(\left(u_{k-1}-u_{k}\right)-\left(u_{k+1}-u_{k+2}\right)\right)+(-1)^{k+1}\left(w_{k}-w_{k+1}\right) \\
& =u_{-k+1}+(-1)^{k+1}\left[\left(w_{k}+\frac{\theta c}{2}\left(w_{k+1}-w_{k-1}\right)+\frac{(1-\theta) c}{2}\left(u_{k+1}-u_{k-1}\right)\right)\right. \\
& \left.-\left(w_{k+1}+\frac{\theta c}{2}\left(w_{k+2}-w_{k}\right)+\frac{(1-\theta) c}{2}\left(u_{k+2}-u_{k}\right)\right)\right] \\
& =u_{-k+1}+(-1)^{k+1}\left(u_{k}-u_{k+1}\right)=u_{-k+1}-\left(u_{-k+1}-u_{-k}\right)=u_{-k},
\end{aligned}
$$

which means that Formula (9) is true for $j=-k$. Formula (9) is proved for $j \in\{-J+1, \ldots, J\}$. 
Using the matrices $P$ and $Q$ defined in the statement, this can be expressed in the form

$$
P w=-Q u+\frac{\theta c}{2}\left(\begin{array}{c}
w_{-J+1}-w_{J} \\
0 \\
\vdots \\
0 \\
w_{-J+1}-w_{J}
\end{array}\right)+\frac{(1-\theta) c}{2}\left(\begin{array}{c}
u_{-J+1}-u_{J} \\
0 \\
\vdots \\
0 \\
u_{-J+1}-u_{J}
\end{array}\right),
$$

which makes $\alpha(u)$ and $\alpha(w)$ appear. Proceeding in the same way in the odd case (we omit the details), we obtain:

$$
P w=-Q u+\frac{\theta c}{2}\left(\begin{array}{c}
w_{-3 J+1}-w_{J} \\
0 \\
\vdots \\
0 \\
w_{-3 J+1}-w_{J}
\end{array}\right)+\frac{(1-\theta) c}{2}\left(\begin{array}{c}
u_{-3 J+1}-u_{J} \\
0 \\
\vdots \\
0 \\
u_{-3 J+1}-u_{J}
\end{array}\right) .
$$

Let us now compare $\alpha(w)$ and $\alpha(u)$.

- If $\mathrm{J}$ is even:

$$
\begin{aligned}
w_{-J+1} & =u_{-J+1}-\frac{\theta c}{2}\left(w_{-J+2}-w_{-J+1}\right)-\frac{(1-\theta) c}{2}\left(u_{-J+2}-u_{-J+1}\right) \\
& =u_{-J+1}-\frac{\theta c}{2}(-1)^{J}\left(w_{J}-w_{J-1}\right)-\frac{(1-\theta) c}{2}(-1)^{J}\left(u_{J}-u_{J-1}\right) \\
& =u_{-J+1}-\frac{\theta c}{2}\left(w_{J}-w_{J-1}\right)-\frac{(1-\theta) c}{2}\left(u_{J}-u_{J-1}\right) \\
& =u_{-J+1}+w_{J}-u_{J} .
\end{aligned}
$$

- If $\mathrm{J}$ is odd:

$$
\begin{aligned}
w_{-3 J+1} & =u_{-3 J+1}-\frac{\theta c}{2}\left(w_{-3 J+2}-w_{-3 J+1}\right)-\frac{(1-\theta) c}{2}\left(u_{-3 J+2}-u_{-3 J+1}\right) \\
& =u_{-3 J+1}-\frac{\theta c}{2}(-1)^{J}(-1)^{J}\left(w_{-2 J+J}-w_{-2 J+J-1}\right)-\frac{(1-\theta) c}{2}(-1)^{J}(-1)^{J}\left(u_{-2 J+J}-u_{-2 J+J-1}\right) \\
& =u_{-3 J+1}-\frac{\theta c}{2}(-1)^{J}(-1)^{2}\left(w_{-J+2}-w_{-J+1}\right)-\frac{(1-\theta) c}{2}(-1)^{J}(-1)^{2}\left(u_{-J+2}-u_{-J+1}\right) \\
& =u_{-3 J+1}-\frac{\theta c}{2}(-1)^{J}(-1)^{J}\left(w_{J}-w_{J-1}\right)-\frac{(1-\theta) c}{2}(-1)^{J}(-1)^{J}\left(u_{J}-u_{J-1}\right) \\
& =u_{-3 J+1}+w_{J}-u_{J}
\end{aligned}
$$

Thus, whatever the parity of $\mathrm{J}, \alpha(w)=\alpha(u)$. From Lemma 3.4 and the linearity of the reconstruction operator, we have

$$
u_{-3 J+j}=u_{-J+1}+u_{J}-u_{-J+j}
$$

for any $j \in\{1, \ldots, J\}$. Thus, taking $j=1$, we obtain

$$
u_{-3 J+1}=u_{-J+1}+u_{J}-u_{-J+1}=u_{J},
$$

which proves that $\alpha(u)=0$ when $J$ is odd. Thus, in any case $w$ satisfies

$$
P w=-Q u+\hat{\alpha}(u) .
$$

As the matrix $P$ is invertible, this proves that $w=v$.

A consequence of the above proposition is that solving Problem (2) (with Neumann conditions) is equivalent to solving the problem

$$
P u^{n+1}=-Q u^{n}+\hat{\alpha}\left(u^{0}\right)
$$

when starting out with $u^{0}=R U^{0}$ being the reconstructed vector for the initial data. The equation is in $\mathbb{R}^{2 J}$ if $J$ is even, and in $\mathbb{R}^{4 J}$ if $J$ is odd. Note that the source term is $\hat{\alpha}\left(u^{0}\right)$ (i.e. time-independent) which is a consequence of the fact that $\alpha(u)=\alpha(w)$ in the proposition. 


\subsection{Step 2 : convergence of the discrete initial condition in $l^{\infty}$}

In this section, we will prove that the discrete reconstruction $u^{0}=R U^{0}$ converges (in $l^{\infty}$ ) toward the

function $u^{0, *}$ which is equal to $u^{0, e}$ if $J$ is even, and to $u^{0, o}$ if $J$ is odd. More precisely, we will prove

Proposition 3.5 Let $u^{0} \in \mathcal{C}_{c}^{2}(0,1)$, let $U_{j}^{0}=u^{0}\left(x_{j}\right)$ for $j=1, \ldots, J,\left(u_{j}\right)_{j \in \mathbb{Z}}=R U^{0}$ and define $e_{j}^{0}=u_{j}^{0}-u^{0, *}\left(x_{j}\right)$. There exists a constant $C$ (depending on the initial data $u^{0}$ ) such that

$$
\left|e_{j}^{0}\right| \leq C \Delta x
$$

for any $j \in\{-J+1, \ldots, J\}$ if $J$ is even, and $j \in\{-3 J+1, \ldots, J\}$ if $J$ is odd.

Proof: When $\mathrm{J}$ is even:

$$
\left\{\begin{array}{l}
e_{j}^{0}=u_{j}^{0} \quad \text { for } j \in\{-J+1, \ldots, 0\} \\
e_{j}^{0}=0 \quad \text { for } j \in\{1, \ldots, J\}
\end{array}\right.
$$

and if $\mathrm{J}$ is odd:

$$
\left\{\begin{array}{l}
e_{j}^{0}=u_{j}^{0} \quad \text { for } j \in\{-3 J+1, \ldots,-2 J\} \\
e_{j}^{0}=u_{j}^{0}+u_{0}\left(x_{j}+2\right) \text { for } j \in\{-2 J+1, \ldots,-J\} \\
e_{j}^{0}=u_{j}^{0} \text { for } j \in\{-J+1, \ldots, 0\} \\
e_{j}^{0}=0 \text { for } j \in\{1, \ldots, J\}
\end{array}\right.
$$

Thanks to lemma 3.4 , if $J$ is odd,

$$
\left\{\begin{array}{l}
e_{j}^{0}=u_{j}^{0} \quad \text { for } j \in\{-3 J+1, \ldots,-2 J\} \\
e_{j}^{0}=u_{-J+1}+u_{J}-u_{J+2 J}+u_{0}\left(x_{j}+2\right) \quad \text { for } j \in\{-2 J+1, \ldots,-J\} \\
e_{j}^{0}=u_{j}^{0} \quad \text { for } j \in\{-J+1, \ldots, 0\} \\
e_{j}^{0}=0 \quad \text { for } j \in\{1, \ldots, J\} .
\end{array}\right.
$$

- For $j \in\{1, \ldots, J\}$, the result is trivial.

- For $j \in\{-J+1, \ldots, 0\}$, we have $j=-J+k$ with $k \in\{1, \ldots, J\}$ and

$$
\begin{aligned}
u_{-J+k}^{0} & =u_{-J+k+1}^{0}-(-1)^{J-k+1}\left(u_{J-k+1}^{0}-u_{J-k}^{0}\right) \\
& =u_{-J+k+2}^{0}-(-1)^{J-k}\left(u_{J-k}^{0}-u_{J-k-1}^{0}\right)-(-1)^{J-k+1}\left(u_{J-k+1}^{0}-u_{J-k}^{0}\right) \\
& =u_{-J+k+2}^{0}+(-1)^{J-k}\left(u_{J-k+1}^{0}-2 u_{J-k}^{0}+u_{J-k-1}^{0}\right),
\end{aligned}
$$

thus $\left|u_{-J+k}^{0}\right| \leq\left|u_{-J+k+2}^{0}\right|+C \Delta x^{2}$ if $u^{0}$ is twice continuously differentiable. By induction, $\left|u_{j}^{0}\right| \leq+C \Delta x$, since $u_{1}^{0}=0$.

- For $j \in\{-2 J+1, \ldots,-J\}$ (if $J$ is odd), we have $j=-2 J+k$ with $k \in\{1, \ldots, J\}$ and, from Lemma 3.4,

$$
u_{-2 J+k}^{0}=u_{-J+1}^{0}+u_{J}^{0}-u_{k}^{0}=u_{-J+1}^{0}-u_{k}^{0}
$$

since $u_{J}^{0}=0$. Thus, as $\left|u_{-J+1}^{0}\right| \leq C \Delta x,\left|e_{j}^{0}\right| \leq C \Delta x$.

- For $j \in\{-3 J+1, \ldots,-2 J\}$, we can repeat the arguments in the second item above.

The result is proved.

\subsection{Step 3 : convergence of $e^{n}$ in $l^{\infty}\left(0, T, l^{2}\right)$}

In this section we show the convergence of $e^{n}$ toward 0 in $l^{\infty}$ in time and $l^{2}$ in space, i.e. Theorem 2.1 .

Lemma 3.6 (stability of the scheme) Scheme 8 is stable in a strong sense, i.e. one has

$$
\left|P^{-1} Q\right|_{2} \leq 1
$$

if and only if $\theta \leq 1 / 2$. 
The lemma expresses the fact that the amplification matrix of the scheme is of $l^{2}$ norm less than 1 (without any condition on $\Delta t$ ).

Proof: Recall that $P=I+\frac{\theta c}{2} T, Q=-I+\frac{(1-\theta) c}{2} T$ with $T$ being the (antisymmetric) matrix from (3). Thus, $\mathrm{P}$ and $\mathrm{Q}$ are normal and commuting so $P^{-1} Q$ is also normal and $\left\|P^{-1} Q\right\| \|_{l^{2}}=$ $\sqrt{\rho\left(\left(P^{-1} Q\right)\left(P^{-1} Q\right)^{*}\right)}=\rho\left(P^{-1} Q\right)$.

Also, $\mathrm{P}$ and $\mathrm{Q}$ are simultaneously diagonalizable. The eigenvalues for $T$ (being antisymmetric), $\tau_{j}$ (for $j \in\{1, \ldots, 2 J\}$ if $J$ is even, and $j \in\{1, \ldots, 4 J\}$ if $J$ is odd) are therefore purely imaginary and the eigenvalues of $P^{-1} Q$ will be given by $\left(-1+\frac{(1-\theta) c}{2} \tau_{j}\right) /\left(1+\frac{\theta c}{2} \tau_{j}\right)$. It follows that $\left(\rho\left(P^{-1} Q\right)\right)^{2}=\sup _{j}\left(1+\frac{(1-\theta)^{2} \tau_{j}^{2} c^{2}}{4}\right) /\left(1+\frac{\theta^{2} \tau_{j}^{2} c^{2}}{4}\right) \leq 1$ if and only if $\theta \geq \frac{1}{2}$. This ends the proof.

We write the evolution formula for the error: $P e^{n+1}=-Q e^{n}+\hat{\alpha}\left(u^{0}\right)+\Delta t \epsilon_{n}$ with $\epsilon_{n}$ being the consistency error of Scheme (8). We may rewrite this as follows:

$$
\begin{aligned}
e^{n+1}=-P^{-1} Q e^{n}+ & P^{-1} \hat{\alpha}\left(u^{0}\right)+\Delta t P^{-1} \epsilon_{n} \\
& =\left(-P^{-1} Q\right)^{n+1} e^{0}+\Delta t \sum_{k=0}^{n}\left(-P^{-1} Q\right)^{n-k} P^{-1} \epsilon_{k}+\sum_{k=0}^{n}\left(-P^{-1} Q\right)^{n-k} P^{-1} \hat{\alpha}\left(u^{0}\right)
\end{aligned}
$$

Thus

$$
\begin{gathered}
\left|e^{n}\right|_{2}=\left|\left(P^{-1} Q\right)^{n} e^{0}\right|_{2}+\sum_{k=0}^{n-1}\left|\left(P^{-1} Q\right)^{n-1-k} P^{-1} \hat{\alpha}\left(u^{0}\right)\right|_{2}+\Delta t \sum_{k=0}^{n-1}\left|\left(P^{-1} Q\right)^{n-1-k} P^{-1} \epsilon_{k}\right|_{2} \\
\leq\left|e^{0}\right|_{2}+\sum_{k=0}^{n-1}\left|\hat{\alpha}\left(u^{0}\right)\right|_{2}+\Delta t \sum_{k=0}^{n-1}\left|\epsilon_{k}\right|_{2}
\end{gathered}
$$

thanks to lemma (3.6), and, finally,

$$
\left|e^{n}\right|_{2} \leq\left|e^{0}\right|_{2}+n \sqrt{2}\left|\alpha\left(u^{0}\right)\right| \Delta x^{\frac{1}{2}}+n \Delta t|| \epsilon \|_{\infty} .
$$

We already know that $\left|e^{0}\right|_{2} \leq\left|e^{0}\right|_{\infty} \underset{\Delta x \rightarrow 0}{\longrightarrow} 0$, thus it remains to show that $n\left|\alpha\left(u^{0}\right)\right| \Delta x^{\frac{1}{2}}$ and $\|\epsilon\|_{\infty}$ converge toward 0 .

We here prove an estimate that is actually finer as in the theorem. We know that the solution of $(6)$ is $u^{*}(t, x)=u^{0, *}(x-c t)$. So, thanks to the assumption $u^{0} \in \mathcal{C}_{c}^{2}(0,1), u^{0, *}$ and $u^{*}$ are of class $\mathcal{C}^{2}(\mathbb{R})$ too, and we have the following consistency estimate :

$$
\begin{aligned}
\left\|\epsilon_{n}\right\|_{\infty} & =\| \frac{u^{*}\left(x_{i}, t_{n+1}\right)-u^{*}\left(x_{i}, t_{n}\right)}{\Delta t}+\theta c \frac{u^{*}\left(x_{i+1}, t_{n+1}\right)-u^{*}\left(x_{i-1}, t_{n+1}\right)}{2 \Delta x} \\
& +(1-\theta) c \frac{u^{*}\left(x_{i+1}, t_{n}\right)-u^{*}\left(x_{i-1}, t_{n}\right)}{2 \Delta x}\left\|_{\infty} \leq C\right\| u_{0}^{\prime \prime \prime} \|_{\infty}(\Delta t+\Delta x) .
\end{aligned}
$$

If moreover we assume $u^{0} \in \mathcal{C}_{c}^{3}(0,1), u^{0, *}$ and $u^{*}$ are of class $\mathcal{C}^{3}(\mathbb{R})$ too, and we have the following more precise consistency estimate :

$$
\begin{aligned}
\left\|\epsilon_{n}\right\|_{\infty} & =\| \frac{u^{*}\left(x_{i}, t_{n+1}\right)-u^{*}\left(x_{i}, t_{n}\right)}{\Delta t}+\theta c \frac{u^{*}\left(x_{i+1}, t_{n+1}\right)-u^{*}\left(x_{i-1}, t_{n+1}\right)}{2 \Delta x} \\
& +(1-\theta) c \frac{u^{*}\left(x_{i+1}, t_{n}\right)-u^{*}\left(x_{i-1}, t_{n}\right)}{2 \Delta x}\left\|_{\infty} \leq C|| u_{0}^{\prime \prime \prime}\right\|_{\infty}\left(|1-2 \theta| \Delta t+\Delta t^{2}+\Delta x^{2}\right) .
\end{aligned}
$$

In the case where $J$ is odd, we have seen that $\alpha\left(u^{0}\right)=0$, thus the result is proved. In the case where $J$ is even we have to bound this quantity. By definition, $\alpha\left(u^{0}\right)=c / 2\left(u_{-J+1}^{0}-u_{J}^{0}\right)=c u_{-J+1}^{0} / 2$ as $u_{J}^{0}=0$, and we have already seen that due to the assumption on the regularity of $u^{0}, u_{-J+1}^{0} \leq C \Delta x$.

Finally, we have the following inequality, valid whatever the parity of $J$ :

$$
\left|e^{n}\right|_{2} \leq C \Delta x+C n \Delta t\left(\Delta t+\Delta x+((J+1) \bmod 2) \Delta x^{1 / 2}\right),
$$

or even, in the case where $u_{0} \in \mathcal{C}_{c}^{3}(0,1)$,

$$
\left|e^{n}\right|_{2} \leq C \Delta x+C n \Delta t\left(|1-2 \theta| \Delta t+\Delta t^{2}+\Delta x^{2}+((J+1) \quad \bmod 2) \Delta x^{1 / 2}\right) .
$$

This proves the theorem. 


\section{Dirichlet boundary condition}

In this section, we take $J \in \mathbb{N}^{*}$, let $\Delta x=1 /(J+1)$ and define the nodes of the grid $y_{j}=j \Delta x$, and we consider the following $\theta$-scheme, that corresponds formally with imposing homogeneous boundary conditions on both sides:

$$
\left\{\begin{array}{l}
\frac{U_{j}^{n+1}-U_{j}^{n}}{\Delta t}+a \theta \frac{U_{j+1}^{n+1}-U_{j-1}^{n+1}}{2 \Delta x}+a(1-\theta) \frac{U_{j+1}^{n}-U_{j-1}^{n}}{2 \Delta x}=0, \quad n \geq 0, \quad 2 \leq j \leq J-1, \\
\frac{U_{1}^{n+1}-U_{1}^{n}}{\Delta t}+a \theta \frac{U_{2}^{n+1}}{2 \Delta x}+a(1-\theta) \frac{U_{2}^{n}}{2 \Delta x}=0, \quad n \geq 0, \\
\frac{U_{J}^{n+1}-U_{J}^{n}}{\Delta t}-a \theta \frac{U_{J-1}^{n+1}}{2 \Delta x}-a(1-\theta) \frac{U_{J-1}^{n+1}}{2 \Delta x}=0, \quad n \geq 0, \\
U_{j}^{0}=U^{0}\left(y_{j}\right), \quad j \in\{1, \ldots, J\} .
\end{array}\right.
$$

Second and third equations above can also be obtained by defining the boundary (fictive) values $U_{0}^{n}=U_{J+1}^{n}=0$, which is consistent with the homogeneous Dirichlet conditions.

Setting $U^{n}=\left(U_{j}^{n}\right)_{j=1}^{J}$, this system also takes the following matrix form:

$$
\bar{A} U^{n+1}+\bar{B} U^{n}=0,
$$

with $\bar{A}=I+\frac{\theta c}{2} \bar{D}, \bar{B}=I+\frac{(1-\theta) c}{2} \bar{D}$ and

$$
\bar{D}=\left(\begin{array}{ccccccc}
0 & 1 & 0 & \cdots & \cdots & \cdots & 0 \\
-1 & 0 & 1 & & & & \vdots \\
0 & -1 & 0 & 1 & & & \vdots \\
\vdots & & \ddots & \ddots & \ddots & & \vdots \\
\vdots & & & & 0 & 1 & 0 \\
\vdots & & & & -1 & 0 & 1 \\
0 & \cdots & \cdots & \cdots & 0 & -1 & 0
\end{array}\right) \in \mathcal{M}_{J}(\mathbb{R})
$$

With these "boundary conditions", we also observe a strange numerical behavior with a periodical in time phenomenon, but with very oscillating intermediate solutions: see Figures 5 and 6 . We can prove a similar theorem. We use the same notations as in Section 2 for $u^{0, e}, u^{0, o}, u^{e}$ and $u^{o}$ and we define

$$
f_{j}^{n}=\left\{\begin{array}{ll}
U_{j}^{n}-u^{o}\left(t^{n}, y_{j}\right) & \text { if } \mathrm{J} \text { is even, } \\
U_{j}^{n}-u^{e}\left(t^{n}, y_{j}\right) & \text { if } \mathrm{J} \text { is odd, }
\end{array} \forall j \in\{0, \ldots, J+1\},\right.
$$

where it is understood that $U_{0}^{n}=0$, and

$$
\tilde{f}_{J}(t, x)=\sum_{j=0}^{J} \sum_{n=0}^{+\infty} f_{j}^{n} \chi_{[j \Delta x,(j+1) \Delta x)}(x) \chi_{[n \Delta t,(n+1) \Delta t)}(t) .
$$

Theorem 4.1 Assume $u^{0} \in \mathcal{C}_{c}^{2}(0,1)$. Then, for any Courant number $c$ and any parameter $\theta \geq 1 / 2$, we have

- (case where $J$ is even) for any $g \in L^{2}(-1,1)$,

$$
\lim _{\Delta x \rightarrow 0}\left\|<u_{2 K}-u^{o}, g>_{L^{2}(-1,1)}\right\|_{L^{\infty}(0, T)}=0
$$

- (case where $J$ is odd) for any $g \in L^{2}(-2,2)$,

$$
\lim _{\Delta x \rightarrow 0}\left\|<u_{2 K+1}-u^{e}, g>_{L^{2}(-2,2)}\right\|_{L^{\infty}(0, T)}=0 .
$$

Here it is understood that, as in the whole paper, $\Delta t=c \Delta x / a$, thus $\Delta t$ tends to 0 at the same velocity as $\Delta x$. 
Observe that the (here weak) limits are not the same as in the Neumann case: the convergence is toward $u^{o}$ as $J$ is even and toward $u^{e}$ when $J$ is odd.

As it is very similar to the proof of the result in the Neumann case, we do not reproduce it here (actually it is even simpler because there is no source terme $\alpha\left(u^{0}\right)$ ). We only give the recontruction operator that has to be used here.

Definition 4.2 The operator $\bar{R}$ is defined in the following way.

If $J$ is odd, $u=\left(u_{-(J+1)}, \ldots, u_{J}\right)=\bar{R} U \in \mathbb{R}^{2(J+1)}$ is the unique vector such that

- $u_{0}=0, u_{-(J+1)}=0$,

- $u_{j}=U_{j}, u_{-j}=(-1)^{j} U_{j}$ for $j \in\{1, \ldots, J\}$.

If $J$ is even, $u=\left(u_{-3(J+1)}, \ldots, u_{J}\right)=\bar{R} U \in \mathbb{R}^{4(J+1)}$, is the unique vector such that

- $u_{0}=0, u_{-(J+1)}=0, u_{-2(J+1)}=0, u_{-3(J+1)}=0$.

- $u_{j}=U_{j}, u_{-j}=(-1)^{j} U_{j}, u_{-(J+1)-j}=(-1)^{j} u_{-(J+1)+j}, u_{-2(J+1)-j}=(-1)^{j} u_{-2(J+1)+j}$ for $j \in\{1, \ldots, J\}$.

Given $u=\bar{R} U$, we define the associated piecewise affine function $u_{\Delta x}$ in the way we defined $f_{\Delta x}$, but here on $[-3,1]$ if $J$ is even, and on $[-1,1]$ if $J$ is odd. Remark that if the initial data is smooth in $(0,1)$, the reconstructed initial function $u_{\Delta x}^{0}$ is oscillating with large amplitude around 0 in $(-1,0)$, and also in $(-3,-2)$ is $J$ if even. More precisely, it is easy to prove that the restriction of $u_{\delta x}^{0}$ to $(-1,0)$ converges weakly to 0 on this interval, and the same for the restriction of $u_{\delta x}^{0}$ to $(-3,-2)$ if $J$ is even. This explains that the convergence is only weak. The rest of the proof follows the line of the preceding analysis (multiplying the error function $f_{\Delta x}$ by an arbitrary funtion in $L^{2}$ ).

\section{$5 \quad$ Numerical results}

In all this section, devoted to a more precise observation of numerical results, the chosen initial condition remains

$$
u_{0}(x)=\left\{\begin{array}{l}
\frac{1}{\exp (-9)} \exp \left(\frac{1}{(x-1 / 2)^{2}-\frac{1}{9}}\right) \text { if } \frac{1}{6}<x<\frac{5}{6} \\
0 \text { sinon }
\end{array}\right.
$$

and we take $a=1$ and $J=1000$ or $J=1001$. All the simulations are done with a Courant number $c=1 / 2$. The numerical results obtained with the implicit scheme $(\theta=1)$ and Neumann conditions has been exposed in section 1 . Here, we zoom in on the numerical solution at time 1 , to see the small oscillations around 0 .
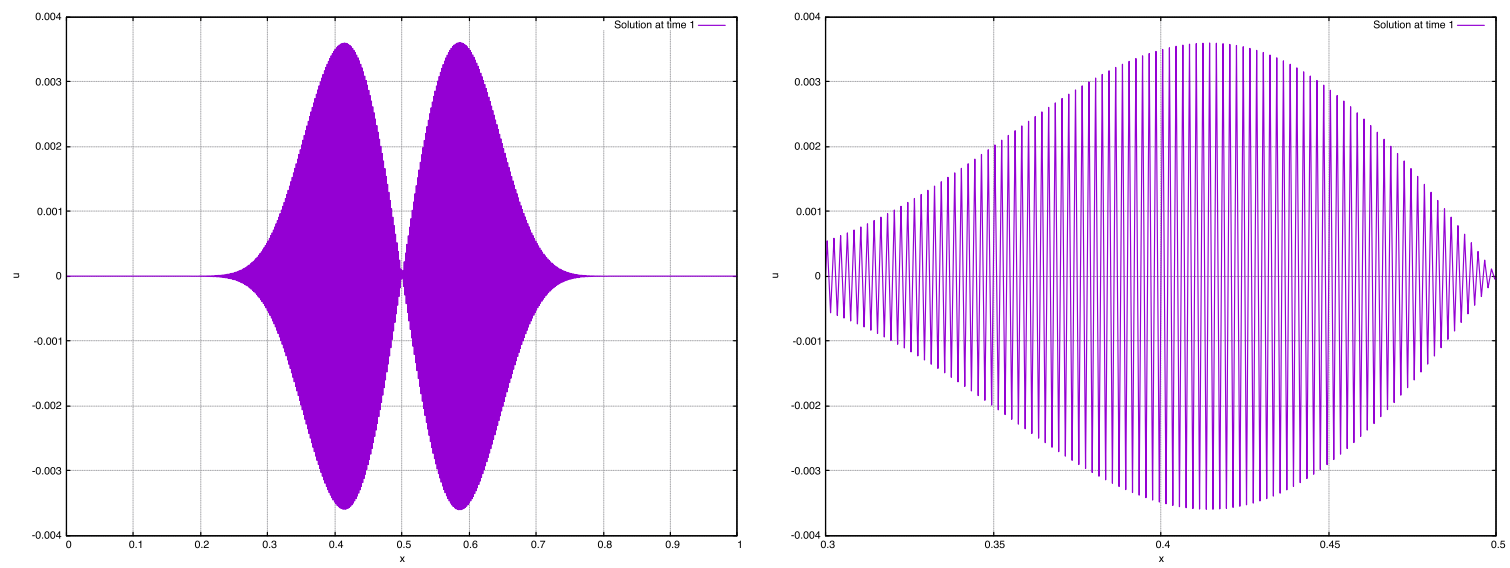

Figure 3: Zoom on the solution at time 1 for $J=1000$ (see the $y$ and $x$ scales).

These small perturbations, which amplitude are of order $\Delta x$ and that are strongly related to the small oscillations of the reconstruted initial data in the proof of the convergence theorem, are moving 
to the left with velocity $-a$. Actually, it is easy to understande this, because if $\left(U_{j}^{n}\right)_{j, n}$ denotes the numerical solution to the implicit centered scheme and is "smooth", then, posing $V_{j}^{n}=(-1)^{j} U_{j}^{n}$ allows to see that

$$
\frac{V_{j}^{n+1}-V_{j}^{n}}{\Delta t}-a \frac{V_{j+1}^{n+1}-V_{j-1}^{n+1}}{2 \Delta x}=0,
$$

that is to say that $V$ has velocity $-a$. This of course remains true for any $\theta \in[1 / 2,1]$. The scheme has a group velocity $-a$ and allow perturbations from the right boundary to go to the left, and when they arrive at position $x=0$, they interact with the left boundary in a "constructive" manner. See [Tre84] for a study of the links between instability and group velocities.

Similar results are observed with $\theta=1 / 2$. However, we note that while diffusion was quite important with the first scheme, it essentially disappears with the Crank Nicolson scheme. We only show the result at time 100 and see that the amplitude remains almost constant.

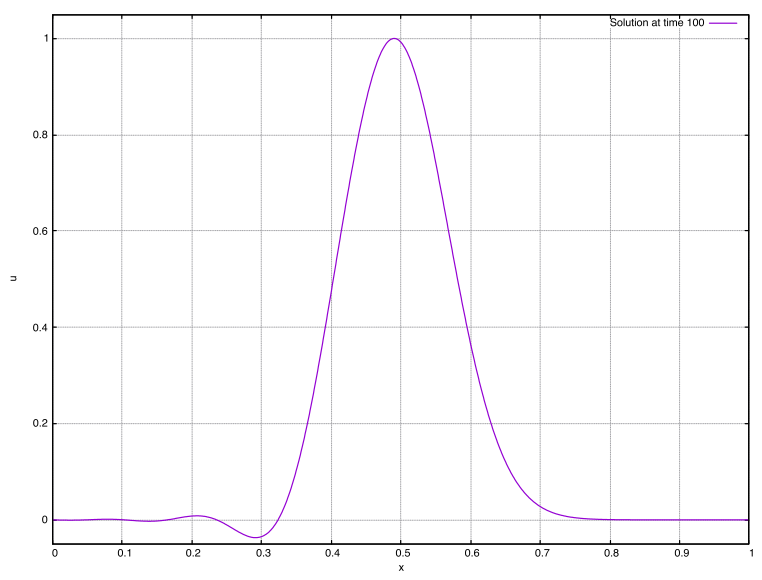

Figure 4: Solution with the Crank-Nicolson scheme $(\theta=1 / 2)$ at time 100 for $J=1000$

At last, we here propose some results obtained with the implicit scheme and Dirichlet boundary conditions, to illustrate Theorem 4.1 

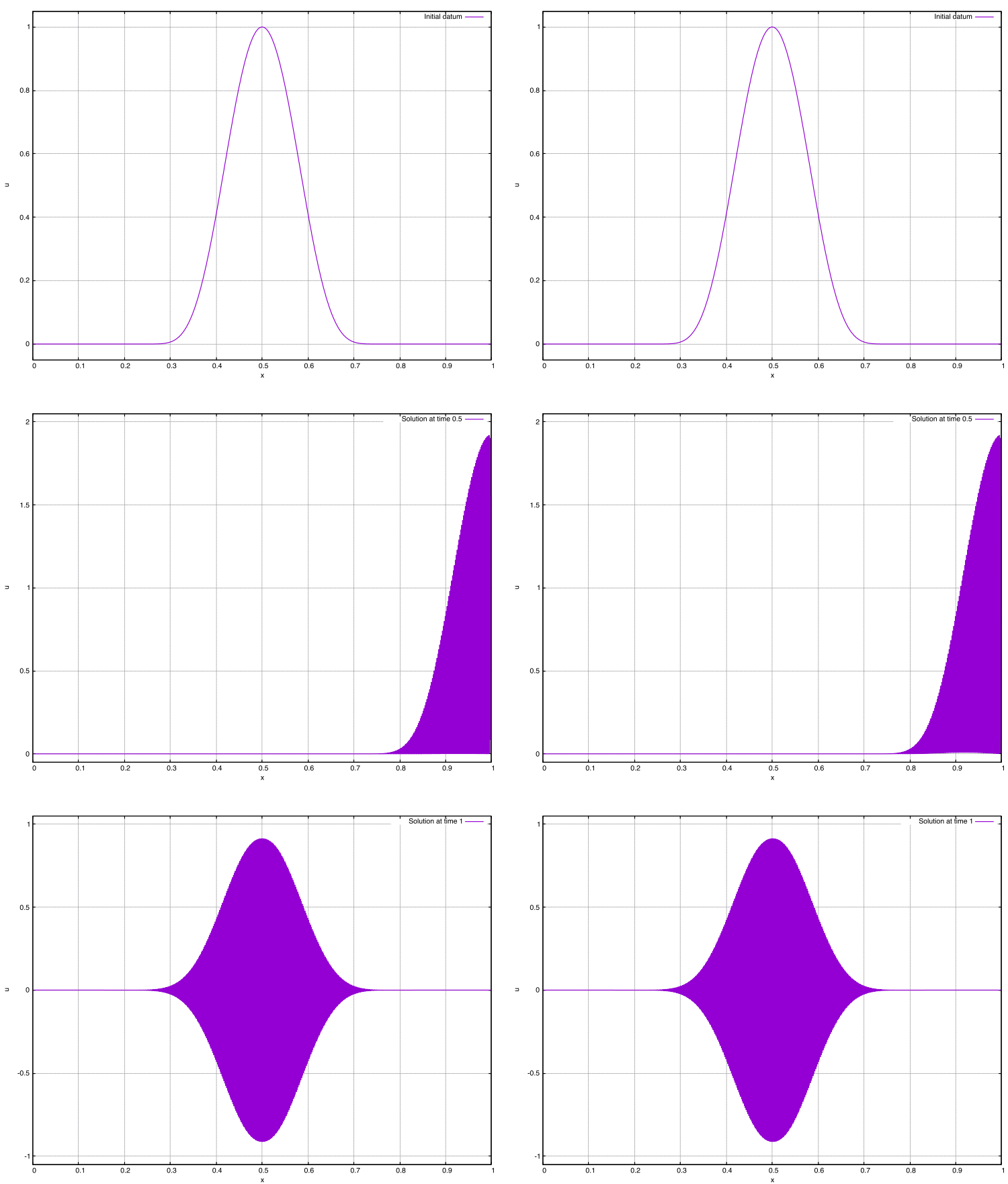

Figure 5: Numerical results: with 1000 cells on the left, 1001 cells on the right. From top to bottom: time $0,0.5,1$. 

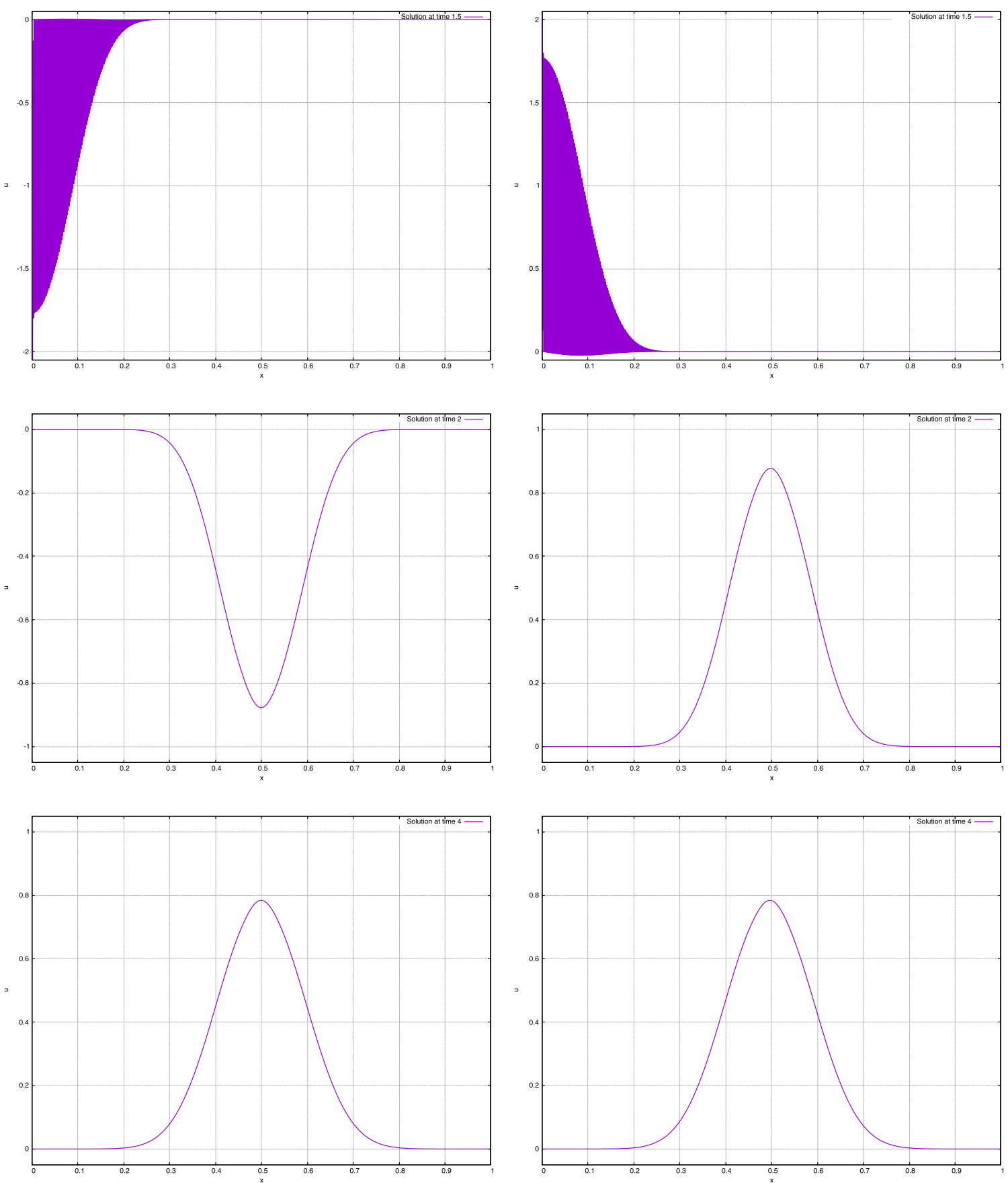

Figure 6: Numerical results: with 1000 cells on the left, 1001 cells on the right. From top to bottom: time $1.5,2,4$.

\section{References}

$\left[\mathrm{AAB}^{+} 08\right]$ X. Antoine, A. Arnold, C. Besse, M. Ehrhardt, and A. Schädle. A review of transparent and artificial boundary conditions techniques for linear and nonlinear Schrödinger equations. Commun. Comput. Phys., 4(4):729-796, 2008.

[BC17] B. Boutin and J.-F. Coulombel. Stability of finite difference schemes for hyperbolic initial boundary value problems: Numerical boundary layers. Numer. Math. Theory Methods Appl., 10(3):489-519, 2017. 
[Ehr10] M. Ehrhardt. Absorbing boundary conditions for hyperbolic systems. Numer. Math. Theory Methods Appl., 3(3):295-337, 2010.

[GKO95] Bertil Gustafsson, Heinz-Otto Kreiss, and Joseph Oliger. Time dependent problems and difference methods. Pure and Applied Mathematics (New York). John Wiley \& Sons, Inc., New York, 1995. A Wiley-Interscience Publication.

[KL68] H.-O. Kreiss and E. Lundqvist. On difference approximations with wrong boundary values. Math. Comp., 22:1-12, 1968.

[Tre84] L. N. Trefethen. Instability of difference models for hyperbolic initial-boundary value problems. Comm. Pure Appl. Math., 37(3):329-367, 1984. 